\title{
Etude des effets vermicide et anti-diarrhéique du macéré aqueux des feuilles de Salvadora persica, L. (Salvadoraceae)
}

\author{
Alfa Bano Halidou GARBA ${ }^{1}$, Moussa AG ARYA ${ }^{1 *}$, Aristide TRAORE ${ }^{2}$ et \\ Sylvin OUEDRAOGO ${ }^{2}$
}

\author{
${ }^{1}$ Université Abdou Moumouni, Faculté des Sciences et Techniques, BP 10662 Niamey, Niger. \\ ${ }^{2}$ Institut de Recherche en Science de la Santé, Département Médecine et Pharmacopée Traditionnelles / \\ Pharmacie, 03 BP 7192 Ouagadougou 03, Burkina Faso. \\ *Auteur correspondant ; E-mail : agarya@yahoo.com ; Tél : (227) 97507243 / 94553557.
}

\section{RESUME}

La présente étude a été réalisée pour évaluer in vitro, les effets vermicides et anti diarrhéiques du macéré aqueux des feuilles de Salvadora persica, L. (Salvadoraceae), plante utilisée au Niger, en médecine traditionnelle, pour le traitement des parasitoses gastro-intestinales. In vitro, le macéré aqueux des feuilles de Salvadora persica, L. a un effet vermicide important sur les vers adultes Ascaridia galli, avec $100 \%$ d'inhibition de leur motilité au bout de 48 heures de contact pour la gamme de concentrations de 1 à $10 \mathrm{mg} / \mathrm{ml}$. Son effet est efficace mais plus lent que le lévamisole $0,01 \%$ qui donne $100 \%$ d'inhibition de motilité, après seulement 2 heures. L'activité contractile normale de l'intestin isolé de lapin, tout comme sa contracture induite par l'acétylcholine $0,35 \mu \mathrm{M}$, ont été inhibées par le macéré aqueux des feuilles de Salvadora persica, L. à $10 \mathrm{mg} / \mathrm{ml}$ montrant ainsi, ses effets antispasmodique et anticholinergique. Le macéré aqueux des feuilles de Salvadora persica, L. aurait des propriétés vermicides, anti diarrhéiques et anticholinergiques et Salvadora persica, L. serait un bon candidat pour la recherche et la mise au point d'un phytomédicament contre la diarrhée.

(C) 2017 International Formulae Group. All rights reserved.

Mots clés : Médecine traditionnelle, Salvadora persica, vermicide, antispasmodique, diarrhée.

\section{Study of the vermicidal and antidiarrheal effects of the aqueous maceration of leaves of Salvadora persica, L. (Salvadoraceae)}

\begin{abstract}
The present study was carried out to evaluate in vitro the vermicidal and antidiarrheal effects of the aqueous maceration of leaves of Salvadora persica L. (Salvadoraceae), a plant used in traditional medicine in Niger for the treatment of gastrointestinal parasitoses. In vitro, the aqueous maceration of leaves of Salvadora persica, L. has a significant vermicidal effect on adult worms Ascaridia galli, with $100 \%$ inhibition of their motility after 48 hours contact for the concentration range of 1 to $10 \mathrm{mg} / \mathrm{ml}$. Its effect is effective but slower than levamisole $0.01 \%$ which gives $100 \%$ inhibition of motility, after only 2 hours. The normal contractile activity of the isolated rabbit intestine, as well as its contracture induced by $0.35 \mu \mathrm{M}$ acetylcholine, were inhibited by the aqueous maceration of Salvadora persica, L. leaves at $10 \mathrm{mg} / \mathrm{ml}$ thus showing its
\end{abstract}


antispasmodic and anticholinergic effects. The aqueous maceration of leaves of Salvadora persica, L. would have vermicidal, anti-diarrheic and anticholinergic properties and Salvadora persica, L. would be a good candidate for the research and development of a phytomedicine for diarrhea.

(C) 2017 International Formulae Group. All rights reserved.

Keywords: Traditional medicine, Salvadora persica, vermicidal, antispasmodic, diarrhea.

\section{INTRODUCTION}

Selon les estimations de l'Organisation Mondiale de la Santé (OMS, 2016), quelques 2,4 milliards de personnes ne disposent toujours pas de toilettes ou de latrines couvertes et le manque d'assainissement est à l'origine de près 280000 décès par maladies diarrhéiques. C'est un facteur majeur dans la propagation de plusieurs maladies tropicales négligées, parmi lesquelles les parasitoses intestinales qui constituent encore de nos jours, un problème de santé publique.

En Afrique, ces affections sont graves, car souvent associées entre elles ou avec une autre maladie parasitaire telle que le paludisme, le tout couronné par la promiscuité, le manque d'eau potable et d'installations sanitaires (Aminata, 2006).

Dans le secteur de l'élevage, l'impact socio-économique de ces parasitoses gastrointestinales se caractérise par d'importantes pertes animales en raison des taux de prévalence des helminthiases pouvant atteindre $98 \%$ dans certaines régions d'élevage (Achi et al., 2003).

Il devient dès lors, indispensable de rechercher dans la riche flore africaine, des plantes médicinales à partir desquelles, seront mises au point, des nouvelles substances efficaces, accessibles, sans toxicité et à large spectre d'action, pour faire face à ces parasitoses.

Salvadora persica $L$. est une plante utilisée depuis plusieurs siècles pour l'hygiène bucco-dentaire. Ses racines et tiges sont traditionnellement utilisées comme brosse dents pour leur donner de l'éclat mais aussi pour lutter contre la mauvaise haleine. Almas (2002) a rapporté plusieurs travaux démontrant ses propriétés antibactériennes, anti- carie et antifongiques. Cette plante est aussi, traditionnellement utilisée dans le traitement des maladies comme l'asthme, la bronchite, la toux, l'infestation par les vers et les hémorroïdes (Noumi et al., 2010). Il est également rapporté que Salvadora persica, L. a des propriétés antibactériennes sur plusieurs souches de bactéries aérobies impliquées dans les infections digestives (Alali et Al-Lafi, 2003); des propriétés anti radicalaires et antiprolifératives (Congo, 2012); antiinflammatoires et antioxydantes (Abhishek et al., 2015 ; Seydou, 2016).

$\mathrm{Au}$ Niger, les feuilles de Salvadora persica, L. sont utilisées par les éleveurs nomades pour traiter les parasitoses internes des animaux (Ag Arya et al., 2008). Cette plante possèderait plusieurs autres propriétés pharmacologiques et à notre connaissance, peu d'investigations ont été faites sur ses effets vermicides et antidiarrhéiques.

Le but de la présente étude est d'évaluer les propriétés vermicides et antidiarrhéiques du macéré aqueux des feuilles de Salvadora persica, L. respectivement, sur Ascaridia galli et l'intestin isolé de lapin afin de contribuer à la validation scientifique de son usage traditionnel.

\section{MATERIEL ET METHODES \\ Matériel \\ Matériel végétal}

Il est constitué de feuilles de Salvadora persica, L. récoltées en juillet 2013 dans la commune urbaine de Tchin-Tabaraden, dans la zone Nord-Ouest de Tahoua, Niger. Après la récolte, les feuilles ont été séchées à l'ombre, à la température $33 \pm 2{ }^{\circ} \mathrm{C}$ et à l'abri de la poussière afin de conserver toutes leurs 
propriétés physico-chimiques. Elles ont ensuite été broyées à l'aide d'un broyeur à lames, pour en faire une fine poudre.

\section{Matériel animal}

Des vers adultes Ascaridia galli sont recueillis à partir des intestins de pintades abattues au marché de volailles de Zogona (Ouagadougou). Ces vers sont maintenus en survie dans une solution physiologique de $\mathrm{NaCl} \quad 0,9 \%$ à la température ambiante de $28 \pm 2{ }^{\circ} \mathrm{C}$, et utilisés pour les tests vermicides.

Des souris de souche Naval Medical Research Institut (NMRI) âgées de 8 semaines, pesant entre 20 et $35 \mathrm{~g}$ ont été utilisées pour l'étude toxicologique. Des lapins pesant $1500 \mathrm{~g}$ en moyenne ont été utilisés pour les études pharmacologiques. Ces animaux sont élevés à l'animalerie de l'Institut de Recherches en Sciences de la Santé (IRSS) de Ouagadougou à une température ambiante de $24 \pm 2{ }^{\circ} \mathrm{C}$ et $60 \%$ d'humidité avec une photopériode de 12 heures. Ils ont accès ad libitum à l'eau et sont nourris à la laitue et/ou tourteau de blé.

\section{Méthodes}

\section{Préparation du macéré aqueux}

Une prise d'essai de $100 \mathrm{~g}$ de poudre de matière végétale a été ajoutée à $1000 \mathrm{ml}$ d'eau distillée dans une boite en verre. Après homogénéisation, le mélange a été laissé au repos pendant 24 heures. Le macéré obtenu a été ensuite filtré sur du tissu nylon à mailles très fines puis, centrifugé à 2000 tours/min pendant 5 minutes. Le surnageant a été recueilli, puis concentré à l'étuve à $50{ }^{\circ} \mathrm{C}$ pendant 24 heures, et lyophilisé. Le lyophilisat obtenu a été conservé dans un flacon et gardé à l'abri de l'humidité et de la lumière.

\section{Préparation des solutions tests et lots expérimentaux pour l'effet vermicide}

Il a été pris $5 \mathrm{ml}$ de la solution de survie $\mathrm{NaCl} 0,9 \%$ dans 5 tubes à essai, on y ajoute le macéré aqueux pour préparer les

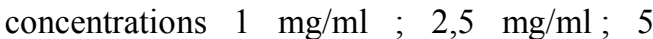

$\mathrm{mg} / \mathrm{ml} ; 7,5 \mathrm{mg} / \mathrm{ml}$ et $10 \mathrm{mg} / \mathrm{ml}$. Ensuite, il a été constitué 5 lots de 5 parasites qui ont été mis en contact avec ces différentes concentrations.

Deux lots de 5 parasites ont été constitués en plus, dont un témoin blanc recevant uniquement le milieu de survie et un témoin de référence recevant une solution de lévamisole $0.01 \%$. Le nombre de morts a été dénombré par lot aux intervalles de temps ciaprès : $2 \mathrm{~h}, 4 \mathrm{~h}, 6 \mathrm{~h}, 12 \mathrm{~h}, 24 \mathrm{~h}, 30 \mathrm{~h}$ et $48 \mathrm{~h}$. Les vers morts s'allongeaient sur toute leur longueur devenant flasques et sans aucune motilité. Le test a été répété trois fois.

Méthode d'étude de la toxicité générale aiguë

La méthode utilisée est celle de la ligne OCDE 432 utilisant un nombre minimum d'animaux par étape et donnant des informations suffisantes sur la toxicité aiguë de la substance pour les besoins de classification. Ainsi 3 souris sont choisies au hasard, marquées et gardées dans des cages individuelles pour les acclimater aux conditions de laboratoire pendant cinq jours avant l'expérience. Ensuite les souris sont mises à jeun pendant 4 heures avant le début de l'expérience, puis il leur a été administré par voie orale, la dose unique de $2000 \mathrm{mg} / \mathrm{kg}$ de poids corporel et observées attentivement pendant les 4 premières heures, et quotidiennement par la suite, pendant 14 jours. L'expérience a été répétée trois fois.

Méthode d'étude de l'activité contractile de l'intestin isolé

Le lapin est préalablement mis à jeun pendant 24 heures avant le début de l'expérience puis anesthésié à l'uréthane $40 \%$. Une fois endormi, l'incision abdominale médiane permet la libération des intestins dont une portion est prélevée et immédiatement mise dans la solution physiologique oxygénée de Tyrode. Cette portion est ensuite découpée en plusieurs fragments de 2 à $4 \mathrm{~cm}$ pour montage et expérimentation dans la cuve à organe isolé thermostatée à $37^{\circ} \mathrm{C}$. L'une des extrémités 
du fragment d'intestin isolé est fixée au crochet de la tige support et l'autre bout, au capteur isométrique qui lui-même est relié à l'enregistreur, par l'intermédiaire d'un amplificateur, qui permet de visualiser les contractions spontanées et régulières de l'intestin isolé après une période de stabilisation de 30 à $45 \mathrm{~min}$ au cours de laquelle, la solution physiologique est renouvelée toutes les $15 \mathrm{~min}$.

Après l'observation de la régularité de l'activité contractile de l'organe isolé, les solutions du macéré aqueux sont administrées en mode cumulatif d'une part, et d'autre part, après la précontracture à l'acétylcholine, en mode curatif, pour évaluer respectivement, l'effet de l'extrait sur l'activité contractile normale de l'intestin isolé et son interaction avec le système cholinergique. L'expérience a été répétée trois fois.

\section{Analyse statistique}

L'analyse statistique des résultats a été réalisée sur la base du traitement statistique du logiciel Graph Prism version 6, One way ANOVA suivi de Dunnett's test. Les valeurs sont présentées sous forme de moyenne \pm erreur standard. Les différences sont considérées significatives si $\mathrm{p}<0,05$ comparativement au témoin.

\section{RESULTATS}

\section{Etude toxicologique}

A l'issue des tests réalisés, après l'administration par voie orale, de la dose unique de $2000 \mathrm{mg} / \mathrm{kg}$ de poids corporel chez les souris, nous n'avons noté ni signe d'intoxication, ni mortalité pendant toute la durée de l'observation. Ce résultat indique que le macéré aqueux des feuilles de Salvadora persica $\mathrm{L}$. a une $\mathrm{DL}_{50}$ qui se situerait dans la catégorie 5 ou non classée $\left(\mathrm{DL}_{50}>5000\right.$ $\mathrm{mg} / \mathrm{kg}$ ), selon le système globalement harmonisé de l'OCDE 423, ce qui le classe dans cette nomenclature, comme produit faiblement toxique. Cette observation corrobore les résultats de Issa et al. (2016) et Seydou (2016).

Des résultats comparables ont été mis en évidence par les travaux de Gome et al. (2011) selon lesquels, aucun comportement anormal, ni décès n'a été constaté chez les animaux traités par voie orale, ce qui n'a pas permis de déterminer une valeur précise de la $\mathrm{DL}_{50}$ qui serait, pour Passiflora foetida, supérieure à $13000 \mathrm{mg} / \mathrm{kg}$ de poids corporel .

\section{Le criblage phytochimique}

La révélation de certaines familles chimiques présentes dans le macéré aqueux des feuilles Salvadora persica, L. a été réalisée grâce aux tests de détection chimique décrits dans les travaux de Ciulei et al. (1982). Le principe de cette méthode est basé sur la capacité des groupes chimiques végétaux à réagir avec certains réactifs chimiques spécifiques ou généraux pour donner des colorations caractéristiques.

Le résultat du criblage phytochimique (Tableau 1) obtenu a permis de noter la présence de quelques groupes chimiques dans le macéré aqueux des feuilles de Salvadora persica, L. Ce sont les glucosides stéroïdiques et triterpéniques, les saponosides, les oses, les coumarines et dérivés, les antracénosides, les polyoses; les flavonoïdes, les tanins et les alcaloïdes. Des résultats similaires ont été mis en évidence, dans les mêmes conditions expérimentales, par les travaux de Issa et al. (2016) et Seydou (2016).

Effet vermicide du macéré aqueux des feuilles de Salvadora persica, L. sur les vers adultes Ascaridia galli

La Figure 1 présente l'effet inhibiteur du macéré aqueux des feuilles de Salvadora persica, L. sur les vers adultes Ascaridia galli. Elle fait ressortir l'inhibition de la motilité des vers après 24 heures, 30 heures et 48 heures de contact avec l'extrait. Ces essais in vitro, montrent que le macéré aqueux des feuilles de Salvadora persica, L. est actif sur Ascaridia galli avec inhibition de 
leur motilité, de 75 à $95 \%$ pour les concentrations de 7,5 et $10 \mathrm{mg} / \mathrm{ml}$ après 24 heures de contact (Figure 1d, e). Après 30 heures de contact avec l'extrait, on enregistre 75 à $95 \%$ d'inhibition (Figure 1a, b) pour les concentrations 1 et $2,5 \mathrm{mg} / \mathrm{ml}$ et 97 à $100 \%$ d'inhibition de motilité (Figure 1c, d, e) pour les concentrations de 5 à $10 \mathrm{mg} / \mathrm{ml}$.

Après 48 heures de contact avec l'extrait, on obtient $100 \%$ d'inhibition de la motilité des vers (Figure 1a, b, c, d, e) pour les concentrations de 1 à $10 \mathrm{mg} / \mathrm{ml}$ avec une différence non significative. Le lévamisol $0,01 \%$ qui est le produit de référence (Figure 1f), donne $100 \%$ d'inhibition de la motilité des vers après seulement 2 heures de contact. Ces résultats montrent que le macéré aqueux des feuilles de Salvadora persica, L. a un effet vermicide significatif sur les vers adultes Ascaridia galli après 48 heures de contact et, comparé au lévamisol qui est le produit de référence, l'extrait est certes actif mais son effet est plus lent que celui du lévamisole.

Les propriétés vermicides de cet extrait seraient liées à la présence de certains groupes chimiques mis en évidence par le criblage phytochimique. Les travaux de Minaflinou Sacca Sidi et al. (2015) ont rapporté que les plantes médicinales doivent leurs propriétés anthelminthiques à leur composition chimique, ce qui corrobore les travaux de Poalini et al. (2003) et Ademola et al. (2009) qui ont rapporté que les tanins, les flavonoïdes et les saponosides jouent un rôle essentiel dans l'activité anthelminthique des plantes.

\section{Effet du macéré aqueux des feuilles de Salvadora persica, L. sur l'activité contractile normale de l'intestin isolé de lapin}

La Figure 2 montre d'une part, l'activité contractile normale de l'intestin isolé de lapin (Figure 2a) et d'autre part, l'effet des concentrations cumulatives de 3 à 10000 $\mu \mathrm{g} / \mathrm{ml} \mathrm{du}$ macéré aqueux des feuilles de
Salvadora persica, L. sur cette même activité (Figure 2b).

Nous observons que le macéré aqueux des feuilles de Salvadora persica, L. ne modifie pas l'activité contractile normale pour les concentrations de 3 à $300 \mu \mathrm{g} / \mathrm{ml}$ mais à partir de $1000 \mu \mathrm{g} / \mathrm{ml}$, on note une légère baisse de la force contractile de l'intestin isolé et cette baisse va se renforcer (- 40\%) pour la concentration $3000 \mu \mathrm{g} / \mathrm{ml}$ pour atteindre une suppression totale de l'activité contractile à la concentration 10000 $\mu \mathrm{g} / \mathrm{ml}$ accompagnée d'une baisse du tonus de base. Ce résultat montre que le macéré aqueux de Salvadora persica, L. possèderait, en mode cumulatif, des propriétés antispasmodiques caractérisées par des effets inotropes et tonotropes négatifs sur l'activité contractile de l'intestin isolé de lapin.

Cet effet antispasmodique dosedépendant traduit une relaxation du muscle lisse intestinal qui serait due, soit à une inhibition de l'influx calcique, soit à une augmentation de l'efflux de calcium sans modification de l'influx ou même à une capture du $\mathrm{Ca}^{2+}$ libre cytoplasmique.

Effet des concentrations croissantes du macéré aqueux des feuilles de Salvadora persica, L. sur la contracture induite par l'acétylcholine $0,35 \mu \mathrm{M}$ (mode curatif)

La Figure 3 représente l'effet des concentrations croissantes du macéré aqueux des feuilles de Salvadora persica, L. après la contracture induite par l'acétylcholine, contracturant de référence sur l'activité contractile de l'intestin isolé.

Nous constatons que sur cet organe isolé, l'acétylcholine induit un effet tonotrope positif important marqué par une contracture avec activité contractile irrégulière de plateau traduisant l'augmentation du péristaltisme du tractus gastro-intestinal. L'ajout des concentrations croissantes de l'extrait, de C1 à $\mathrm{C} 5 \quad(3$ à $300 \mu \mathrm{g} / \mathrm{ml})$ ne modifie pas significativement le niveau du plateau de contracture et à partir de $\mathrm{C} 6(1000 \mu \mathrm{g} / \mathrm{ml})$, on 
enregistre une baisse de près de $35 \%$ et cette diminution va se renforcer à la concentration C7 (- 65\%) pour atteindre $100 \%$ de suppression de la contracture provoquée par l'acétylcholine.

Cette expérience en mode curatif montre que le macéré aqueux des feuilles de Salvadora persica, L. possède des propriétés anticholinergiques. Cet effet s'expliquerait probablement par la présence dans l'extrait, de certains groupes chimiques qui s'opposent aux actions de l'acétylcholine.

\section{Effet de l'acétylcholine $0,35 \mu M \quad$ sur l'action antispasmodique des concentrations uniques du macéré aqueux des feuilles de Salvadora persica, L. (mode préventif)}

La Figure 4 permet d'évaluer l'action tonotrope et contracturante de l'acétylcholine précédée par l'effet antispasmodique obtenu, suite à l'administration des doses uniques du macéré. Cette expérience en mode préventif, montre que les doses uniques du macéré provoquent un effet antispasmodique important variant de 72 à $100 \%$ pour les concentrations 300 à $10000 \mu \mathrm{g} / \mathrm{ml}$ (Figure 4a, b, c, d). Après cet effet, l'ajout de l'acétylcholine, provoque un effet tonotrope positif mais réduit de $26 \%$ par rapport à celui induit par action directe de l'acétylcholine (mode curatif précédent), marqué par un plateau avec activité contractile irrégulière (Figure 4a, b). La diminution de l'effet tonotrope positif dû à l'acétylcholine, se renforce pour atteindre 80 à $100 \%$ avec inhibition de l'activité contractile de plateau (Figure 4c,d).

Ce résultat, ajouté à celui de la Figure 3 , montre que le macéré aqueux des feuilles de Salvadora persica, L. exercerait une inhibition de type compétitif avec l'acétylcholine au niveau de ses récepteurs sur le muscle lisse intestinal du lapin.

Tableau 1 : Groupes chimiques mis en évidence dans le macéré aqueux des feuilles de Salvadora persica, L.

\begin{tabular}{lc}
\hline Groupes chimiques & Résultats \\
\hline Glucosides stéroïdiques et triterpéniques & + \\
Polyoses & + \\
Tanins & + \\
Oses & + \\
Coumarines et dérivés & + \\
Flavonoïdes & + \\
Antracénosides & + \\
Les alcaloïdes sels & + \\
Saponosides &
\end{tabular}




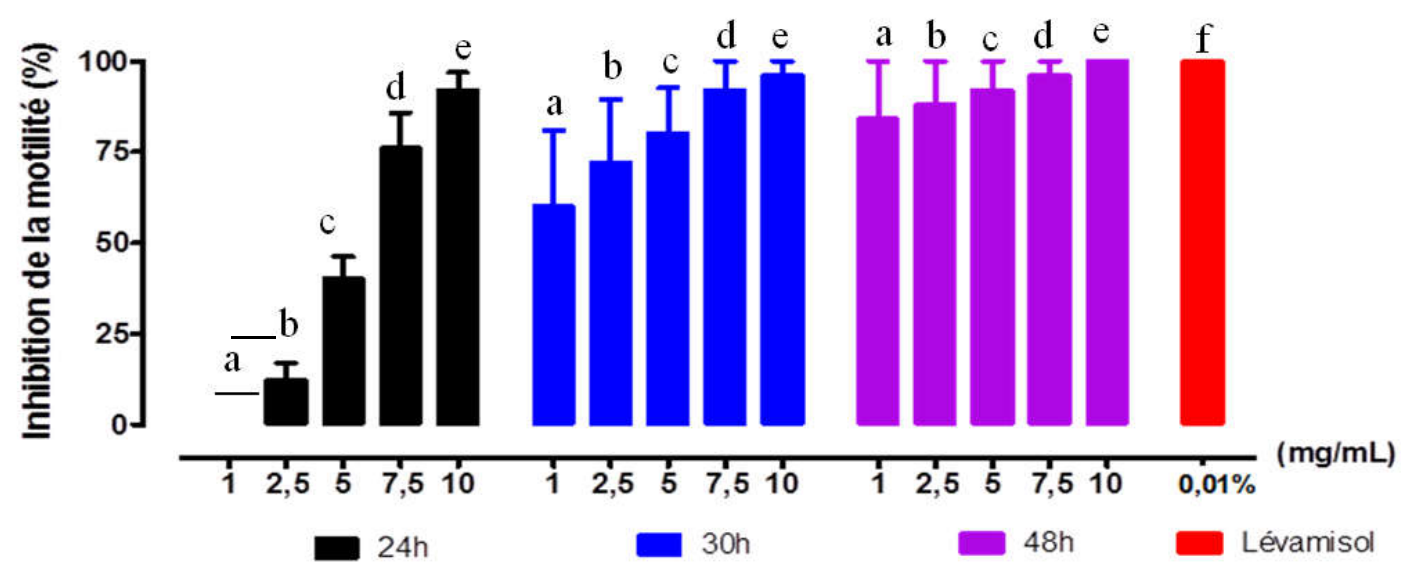

Figure 1: Histogrammes de l'inhibition de la motilité des vers adultes Ascaridia galli en fonction de la dose et de la durée de contact avec le macéré aqueux de Salvadora persica, L.

Les valeurs sont des moyennes \pm erreurs standards $(\mathrm{p}<0,05)$.

L'inhibition de la motilité des vers est obtenue avec le macéré après 48 heures alors que pour le lévamisole, le produit de référence, elle est obtenue après 2 heures.

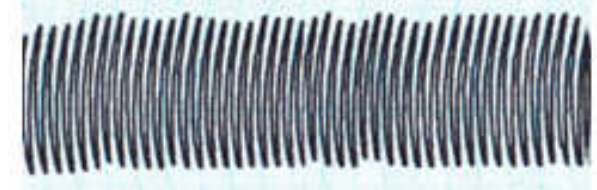

a.

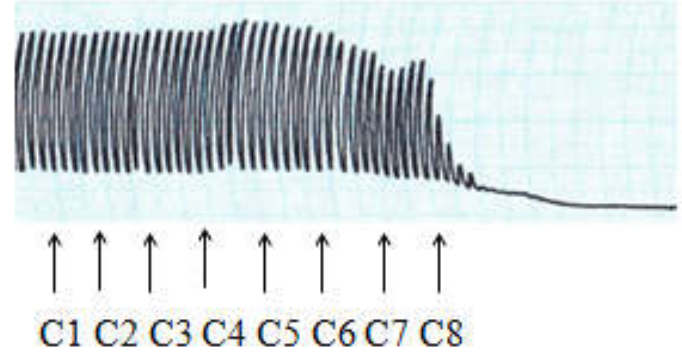

b.

Figure 2 : Activité contractile normale de l'intestin isolé de lapin (a) et effet des concentrations cumulatives $(\mathrm{C} 1$ à $\mathrm{C} 8)$ du macéré aqueux des feuilles de Salvadora persica, L. sur l'activité contractile normale (b).

$\mathrm{C}_{1}=3 \mu \mathrm{g} / \mathrm{ml} ; \mathrm{C}_{2}=10 \mu \mathrm{g} / \mathrm{ml} ; \mathrm{C}_{3}=30 \mu \mathrm{g} / \mathrm{ml} ; \mathrm{C}_{4}=100 \mu \mathrm{g} / \mathrm{ml} ; \mathrm{C}_{5}=300 \mu \mathrm{g} / \mathrm{ml}$,

$\mathrm{C}_{6}=1000 \mu \mathrm{g} / \mathrm{ml} ; \mathrm{C}_{7}=3000 \mu \mathrm{g} / \mathrm{ml} ; \mathrm{C} 8=10000 \mu \mathrm{g} / \mathrm{ml}$.

Le macéré provoque un effet antispasmodique sur l'activité contractile normale à partir de $1000 \mu \mathrm{g} / \mathrm{ml}$ qui devient plus marqué pour la concentration $10000 \mu \mathrm{g} / \mathrm{ml}$. 


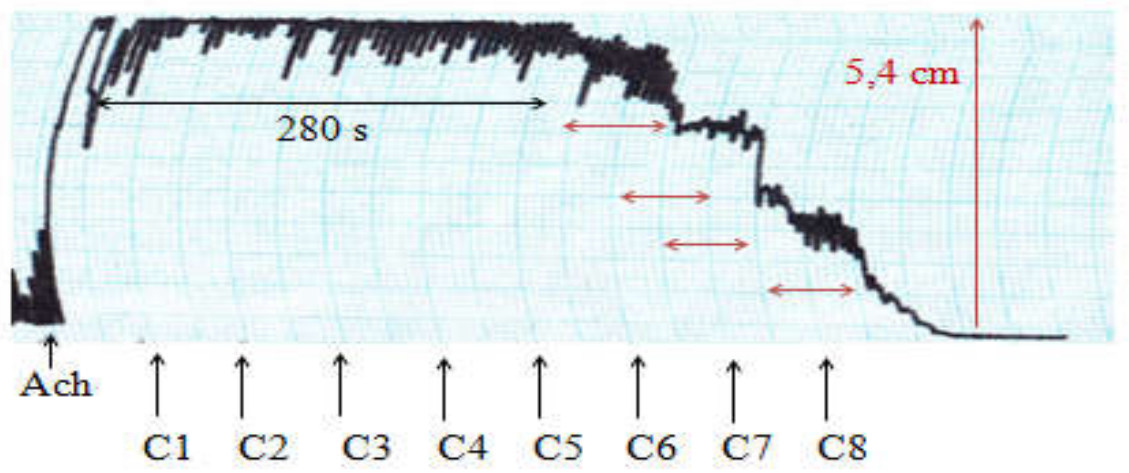

Figure 3 : Effet des concentrations croissantes (mode curatif) du macéré aqueux des feuilles de Salvadora persica, L. après la contracture provoquée par l'acétylcholine. 0,35 $\mu \mathrm{M}$ sur l'activité contractile de l'intestin isolé de lapin.

$\mathrm{C}_{1}=3 \mu \mathrm{g} / \mathrm{ml} ; \mathrm{C}_{2}=10 \mu \mathrm{g} / \mathrm{ml} ; \mathrm{C}_{3}=30 \mu \mathrm{g} / \mathrm{ml} ; \mathrm{C}_{4}=100 \mu \mathrm{g} / \mathrm{ml} ; \mathrm{C}_{5}=300 \mu \mathrm{g} / \mathrm{ml}$,

$\mathrm{C}_{6}=1000 \mu \mathrm{g} / \mathrm{ml} ; \mathrm{C}_{7}=3000 \mu \mathrm{g} / \mathrm{ml} ; \mathrm{C} 8=10000 \mu \mathrm{g} / \mathrm{ml}$; Ach= acétylcholine

L'acétylcholine induit une augmentation du tonus et une contracture sur l'intestin isolé. Cet effet a été d'abord réduit puis inhibé par les concentrations croissantes du macéré.
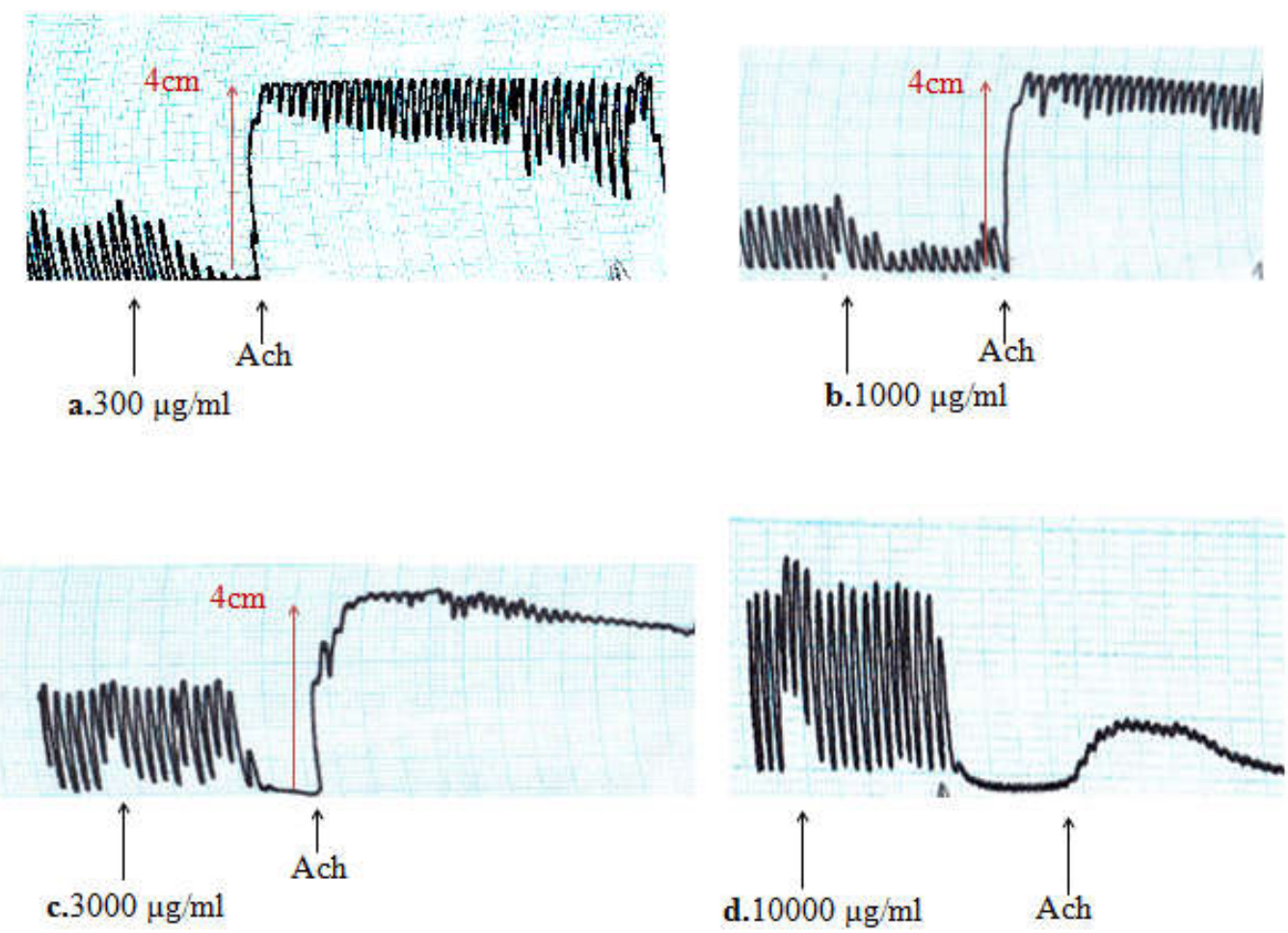

Figure 4 : Effet de l'acétylcholine $0,35 \mu \mathrm{M}$ sur l'action antispasmodique des concentrations uniques du macéré aqueux des feuilles de Salvadora persica, L. (mode préventif). Ach : acétylcholin. L'acétylcholine provoque un effet tonotrope positif mais réduit de $26 \%$ par rapport à son action directe sur l'intestin isolé et une contracture avec activité contractile de plateau (a,b). Cet effet de l'acétylcholine, est d'abord diminué puis inhibé en présence du macéré (c, d). 


\section{DISCUSSION}

L'effet vermicide du macéré aqueux des feuilles de Salvadora persisca, L., sur Ascaridia galli est obtenu après 24 heures de contact pour les doses de 7,5 et $10 \mathrm{mg} / \mathrm{ml}$ et après 48 heures, les concentrations de 1 à 10 $\mathrm{mg} / \mathrm{ml}$ ont donné $100 \%$ d'inhibition de la motilité des vers. Le lévamisol $0,01 \%$ qui est le produit de référence, donne 100\% d'inhibition de la motilité des vers après seulement 2 heures de contact. Ces résultats montrent que le macéré aqueux des feuilles de Salvadora persica, L. a un effet vermicide significatif sur les vers adultes Ascaridia galli après 48 heures de contact et, comparé au lévamisol qui est le produit de référence, l'extrait est certes actif mais son effet est plus lent que celui du lévamisole. Minaflinou Sacca Sidi et al. (2015) ont rapporté que les plantes médicinales doivent leurs propriétés anthelminthiques à leur composition chimique.

Le criblage phytochimique du macéré aqueux des feuilles de Salvadora persisca, L. a montré qu'il contient des saponosides, des flavonoïdes, des alcaloïdes et tanins qui sont bien connus pour leurs propriétés antimicrobiennes (Hoekou et al., 2016) et leur présence serait responsable des propriétés anti parasitaires (Hoste et al., 2006; MarieMagdeleine et al., 2009; Hay et al., 2009) et anti diarrhéiques (Hoekou et al., 2012; Kouadio et al., 2013) . En effet, selon Poalini et al. (2003), les tanins et les flavonoïdes jouent un rôle essentiel dans l'activité anthelminthique des plantes.

Les saponosides auraient un effet anthelmintique par déstabilisation des membranes et augmentation de la perméabilité des cellules, ce qui va entrainer la turgescence des cellules et leur éclatement (McAllister et al., 2001 ; Aharoni et al., 2005 ; Ademola et al., 2009).
Les propriétés vermicides de l'extrait aqueux des feuilles de Salvadora persisca, L. sont à rapprocher de celles mises en évidence par les travaux de Belemlilga (2012) qui ont montré que l'extrait aqueux des feuilles de Saba senegalensis était efficace sur les vers adultes de Haemonchus contortus. Des résultats comparables ont été obtenus sur les vers adultes de Haemonchus contortus par Marie-Magdeleine et al. (2009) avec l'extrait méthanolique de l'écorce de Cucurbita moschata et Hounzangbé-Adoté (2004) avec les extraits éthanoliques de Zanthoxylum zanthoxyloïdes et Newbouldia laevis.

L'étude des effets du macéré aqueux des feuilles de Salvadora persica, L. sur l'activité contractile de l'intestin isolé de lapin a montré qu'il a effet antispasmodique significatif à partir de la dose $1000 \mu \mathrm{g} / \mathrm{ml}$, il devient plus prononcé pour les doses 3000 et $10000 \mu \mathrm{g} / \mathrm{ml}$.

Sanders (2001) rapporte que dans les cellules musculaires lisses, l'augmentation cytoplasmique de la concentration de calcium est le stimulus primaire pour la contraction, qui est généralement le résultat à la fois de la libération intracellulaire du calcium stocké et l'afflux de calcium extracellulaire. L'effet antispasmodique dose-dépendant du macéré aqueux de feuilles de Salvadora persica, $L$. sur l'activité contractile de l'intestin isolé pourrait être la conséquence d'une capture du calcium par des protéines phosphorylées sous l'influence d'une protéine kinase activée par l'AMPc, ou d'une inhibition de l'influx de calcium ou d'un accroissement de l'efflux de calcium sans modification de l'influx, provoquant la vasodilatation et la relaxation des muscles lisses gastro-intestinaux et bronchiques comme le rapporte Schmitt (1989).

L'effet antispasmodique de l'extrait montre qu'il possède des propriétés anti diarrhéiques et il agirait en déprimant le 
péristaltisme du muscle lisse intestinal par diminution de la concentration $\mathrm{du} \mathrm{Ca}^{2+}$ libre cytoplasmique. Les propriétés anti diarrhéiques de l'extrait seraient certainement liées à la présence de certains groupes chimiques dont les tanins, les flavonoïdes, les saponosides connus pour leurs propriétés anti diarrhéiques comme l'ont rapporté les travaux de Hoekou et al. (2012), Kouadio et al. (2013), Minaflinou Sacca Sidi et al. (2015), et Hoekou et al. (2016).

Nous n'avons pas connaissance, des travaux réalisés sur les propriétés anti diarrhéiques des feuilles de Salvadora persica, L. et les résultats obtenus lors de nos tests supportent l'existence des potentialités antidiarrhéiques du macéré aqueux des feuilles de Salvadora persica, L. ce qui justifierait probablement son utilisation traditionnelle pour traiter les parasitoses internes des animaux chez les éleveurs nomades du Niger (Ag Arya et al., 2008).

L'interaction entre l'acétylcholine et le macéré aqueux des feuilles de Salvadora persica, L. aussi bien en mode curatif qu'en mode préventif, mise en évidence par nos résultats, sur l'activité contractile de l'intestin isolé de lapin, montre que cet extrait aurait des propriétés anticholinergiques dosedépendantes.

La contraction induite par l'acétylcholine est liée à l'activation des récepteurs muscariniques M3 sur le muscle lisse couplés aux protéines $\mathrm{G}$ entraînant, via l'Inositol Triphosphate (IP3), la libération de $\mathrm{Ca}^{2+}$ intracellulaire (Gerthoffer, 2005 ; Unno et al., 2005).

L'effet anticholinergique du macéré aqueux des feuilles de Salvadora persica, L. sur l'intestin isolé de lapin s'expliquerait par le fait qu'il contiendrait des substances antagonistes (directes ou indirectes) de l'acétylcholine responsables de l'activité anticholinergique en agissant sur les récepteurs M3 (Belemlilga, 2012). Cette propriété anticholinergique pourrait être liée à la présence dans le macéré aqueux, de certains groupes chimiques dont les alcaloïdes, les coumarines et dérivés, les saponosides et tanins (Poalini et al., 2003 ; Hoste et al., 2006).

\section{Conclusion}

L'étude réalisée in vitro, avec le macéré aqueux des feuilles de Salvadora persica, L. a permis de montrer qu'il a des propriétés vermicides, anti diarrhéiques et anticholinergiques qui s'expliqueraient par sa riche composition chimique. L'effet antispasmodique qu'il exerce sur l'activité contractile du muscle lisse intestinal couplé aux interactions mises en évidence avec l'acétylcholine, lui conférerait des propriétés antidiarrhéiques, ce qui plaiderait en faveur de son utilisation traditionnelle contre la diarrhée. Des investigations poussées sur les propriétés antiparasitaires des feuilles de Salvadora persica, L. permettraient de mieux cerner leurs spécificités thérapeutiques et pourraient déboucher sur la mise au point d'un phytomédicament pour la validation scientifique de son utilisation traditionnelle dans le traitement de la diarrhée, ce qui contribuerait à la dynamique de valorisation des plantes médicinales africaines.

\section{CONFLIT D'INTERETS}

Les auteurs de la présente étude déclarent qu'il n'y a aucun conflit d'intérêts et ce travail constitue l'illustration parfaite d'un partenariat fructueux Sud-Sud entre l'IRSS de Ouagadougou et la Faculté des Sciences et Techniques de l'Université A.M. de Niamey.

\section{CONTRIBUTION DES AUTEURS}

ABHG est l'investigateur principal; MAA et AT ont participé activement à toutes les phases de l'étude; SO a supervisé tous les 
travaux. Tous les auteurs ont lu et approuvé le manuscrit.

\section{REMERCIEMENTS}

Nous adressons nos vifs remerciements au personnel administratif et technique de l'IRSS de Ouagadougou pour tout le soutien technique et financier apporté dans le cadre du présent travail.

\section{REFERENCES}

Abhishek G, Shikhar V, Pradeep K, Sharad S, Rawat AKS. 2005. Phytochemical and antioxidant studies of Salvadora persica, L. Stem \& Twig. Indian Journal of Pharmaceutical Education and Research, 49:71-75. DOI : 10.5530/ijper.49.1.10

Achi YL, Zinsstag J, Yeo N, Dea V, Dorchies PH. 2003. Epidémiologie des helminthoses des moutons et des chèvres dans la région des savanes du Nord de la Côte d'Ivoire. Revue de Médecine Vétérinaire, 154: 179188.

Ademola IO, Eloff JN. 2009. In vitro anthelmintic activity of Combretum molle (R. Br. ex G. Don) (Combretaceae) against Haemonchus contortus ova and larvae. Veterinary Parasitology, 169: 198-203. DOI:http://dx.doi.org/10.1016/j.vetpar.200 9.12 .036

Ag Arya M, Salla A, Ilhidji El Ha, Bangana I. 2008. Maladies parasitaires animales, réponses thérapeutiques de l'Ethnopharmacopée vétérinaire de la zone pastorale de Tahoua/Niger. Pharm. Méd. Trad. Afr., 15: 36-40.

Aharoni A, Jongsma MA, Bouwmeester HJ. 2005. Volatile science metabolic engineering of terpenoids in plants. Trends in Plant Science, 10: 594-602. DOI: http://dx.doi.org/10.1016/j.tplants.2005.10. 005

Almas K. 2002. The Effect of Salvadora Persica Extract (Miswak) and
Chlorhexidine Gluconate on Human Dentin: A SEM Study. J. Contemp. Dent. Pract., 3(3): 027-035.

Alali F, Al-Lafi T. 2003. GC-MS Analysis and bioactivity testing of volatile oil from the leaves of the toothbrush tree Salvadora persica L. Nat Prod Res., 17(3): 189-194. DOI:http://dx.doi.org/10.1080/105756302 1000040790

Aminata N. 2006. Contribution à l'étude des parasitoses Intestinales à l'Institut de pédiatrie sociale de Pikine Guédiawaye. Thèse Doctorat d'Etat en Pharmacie, Université Cheikh Anta Diop de Dakar, 78 p.

Belemlilga BM. 2012. Etudes in vitro des propriétés anthelminthiques du décocté aqueux des feuilles de Saba senegalensis (ADC) Pichon (Apocynaceae), plante utilisée en médecine traditionnelle au Burkina Faso. Mémoire Master, Université de Ouagadougou, 118 p.

Ciulei I. 1982. Practical manuals on the industrial utilization of chemical and aromatic plants. Methodology for analysis of vegetable drugs. Ed. Ministry of chemical industry, Bucharest, p. 67.

Congo MYM. 2012. Etude des propriétés Antiradicalaire et Antiproliférative $\mathrm{d}^{\text {ee }}$ extraits de feuilles et rameaux de Salvadora persica, L. Thèse Doctorat en Pharmacie, Université de Ouagadougou, $120 \mathrm{p}$.

Gerthoffer WT. 2005. Signal-transduction pathway that regulate visceral smooth muscle function III. Coupling of muscarinic receptors to signaling kinases and effector proteins in gastrointestinal smooth muscle. American Journal of Physiology- Gastrointestinal and Liver Physiology, 288(2): G849-G853. DOI: 10.1152/ajpgi.00530.2004

Gome MB, Kouakou K, Toure A, Traore F. 2011. Étude de la toxicité aiguë et 
subchronique de l'extrait aqueux de Passiflora foetida Linn.(Passifloraceae) chez les rats et les souris. Int. J. Biol. Chem. Sci., 5(5): 1777-1789. DOI: http://dx.doi.org/10.4314/ijbcs.v5i5.1

Hay AE, Merza J, Landreau A, Litaudon M, Pagniez F, Le Pape P, Richomme P. 2008. Antileishmanial polyphenols from Garcina vieillardii. Fitoterapia, 79(1): 42-46. DOI: http://dx.doi.org/10.1016/j.fitote.2007.07.0 05

Houekou YP, Tchacondo T, Gbogbo AK, Agban A, Pissang P, Atakpama W, Karou SD, Batawila K et Akpagana K. 2016. Activités antimocrobiennes de Parquetina nigrescens (Afzel.) Bullock, une plante utilisée en médecine traditionnelle togolaise dans le traitement des infections microbiennes. Afrique Science, 12(5): 182188. http://www.afriquescience.info

Hoekou YP, Batawila K, Gbogbo KA, Karou DS, Ameyapoh Y, Souza C. 2012. Evaluation des propriétés antimicrobiennes de quatre plantes de la flore togolaise utilisées en médecine traditionnelle dans le traitement des diarrhées infantiles. Int. J. Biol. Chem. Sci., 6(6): 3089-3097. DOI: http://dx.doi.org/10.4314/ijbcs.v6i6.10

Hounzangbé-Adoté MS. 2004. Propriétés anthelminthiques de 4 plantes tropicales testées in vitro et in vivo sur les nématodes gastro-intestinaux chez les petits ruminants Djallonké. Thèse de doctorat es sciences, FAST- Université d'Abomey-Calavi, p. 205.

Hoste H, Jackson F, Athanasiadou S, Thamsborg SM, Hoskin SO. 2006. The effects of tannin-rich plants on parasitic nematodes in ruminants. Trends in Parasitology, 22(6): 253-261. DOI: http://dx.doi.org/10.1016/j.pt.2006.04.004

Issa I, Ag Arya M, Traoré A, Ouedraogo GG, Gréma M, Ouedraogo S. 2016. Etude des effets bronchodilatateurs des macérés aqueux et hydroéthanolique des feuilles de Salvadora persica L. (Salvadoraceae) sur la trachée isolée de lapin. Annales de l'Université Abdou Moumouni, 2(Tome XXI-A): 82-88.

Kouadio FK, Guessennd NK, Karamoko O, Bahi C, Adama C, Dosso M. 2013. Action antibactérienne de l'extrait éthanolique $70 \%$ de Clerodendrum splendens (G. Don) (Verbenacae) sur des souches bactériennes isolées de selles chez des enfants diarrhéiques. Int. J. Biol. Chem. Sci., 7(3): 1332-1337.

DOI

http://dx.doi.org/10.4314/ijbcs.v7i3.38

Marie-Magdeleine C, Hoste H, Mahieu M, Varo H, Archimede H. 2009. In vitro effects of Cucurbita moschata seed extracts on Haemonchus contortus. Veterinary Parasitology, 161: 99-105. DOI:10.1016/j.vetpar.2008.12.008

Minaflinou Sacca Sidi IY, Azando EVB, Olounlade PA, Hounzangbe-Adote MS. 2015. Effets combinés des feuilles de Newbouldia laevis et de Zanthoxylum zanthoxyloïdes sur les nématodes parasites gastro-intestinaux des ovins Djallonké. Int. J. Biol. Chem. Sci., 9(4) : 2078-2090. DOI: http://dx.doi.org/10.4314/ijbcs.v9i4.30

McAllister TA, Annett CB, Cockwill CL, Olson ME, Wang Y, Cheeke PR. 2001. Studies on the use of Yucca schidigera to control giardiosis. Vet Parasitol., 97: 8599. DOI: $10.1007 / \mathrm{s} 11101-005-1234-5$

Noumi E, Snoussi M, Hajlaoui H, Valentin E, Bakhrouf A. 2010. Antifungal properties of Salvadora persica and Juglans regia L. extracts against oral Candida strains. Eur $J$ Clin Microbiol Infect Dis., 29(1): 81-88. DOI: 10.1007/s10096-009-0824-3.

OCDE. 2001. Guideline for testing of chemicals. Essai $\mathrm{N}^{\mathrm{O}} 423$

OMS. 2016. Centre des Médias. Assainissement. Aide-mémoire $\mathrm{N}^{\circ} 391$ 
Poalini V, Bergeaud JP, Grisez C, Prevot F, Dorchies P, Hoste H. 2003. Effects of condensed tannins on goats experimentally infected with Haemonchus contortus .Vet. Parasitol., 113(3-4) : 253-261. DOI : http://dx.doi.org/10.1016/s03044017(03)00064-5

Seydou S. 2016. Etude des proprieties antiinflammatoire et anti-oxydante des macérés aqueux et hydroéthanoliques des feuilles de Salvadora persica, L. (Salvadoraceae). Mémoire Master, Université Abdou Moumouni, Niamey, $74 \mathrm{p}$.

Sanders KM. 2001. Invited review: mechanisms of calcium handling in smooth muscles. Journal of Applied Physiology, 91: 1438-1449. DOI: http://www.jap.org

Schmitt H. 1989. Interférence avec le calcium. In Eléments de Pharmacologie ( $7^{\grave{e}}$ édn). Flammarion Médecine-Sciences: Paris; 58-60.

Unno T, Matsuyama $\mathrm{H}$, Sakamoto $\mathrm{T}$, Uchiyama $\mathrm{M}$, Izumi $\mathrm{Y}$, Okamoto $\mathrm{H}$, Yamada M, Wess J, Komori S. 2005. M2 and M3 muscarinic receptor-mediated contractions in longitudinal smooth muscle of the ileum studied with receptor knockout mice. British Journal of Pharmacology, 146: 98-108. DOI: 10.1038/sj.bjp.0706300 